\title{
Coupled Resonator Optical Waveguides
}

\author{
Shayan Mookherjea, Student Member, IEEE and Amnon Yariv, Life Fellow, IEEE
}

\begin{abstract}
Properties of the recently introduced family of coupled resonator optical waveguides (CROWs) are reviewed, particularly with reference to CROWs designed as planar waveguides in two-dimensional photonic crystal slabs to enhance nonlinear interactions and develop novel all-optical information processing devices. Topics covered include: pulse propagation both in the nondispersive approximation and to all orders of dispersion, and the coupled mode theory of nonlinear optics with pulses in CROWs and its applications to second-harmonic generation and wave coupling via field-induced refractive-index gratings. We also review recent experimental progress in the fabrication and characterization of CROWs, and applications of the CROW concept to fiber gratings and microwave waveguides.
\end{abstract}

Index Terms-Nonlinear optics, photonic crystals, picosecond phenomena, waveguides.

\section{INTRODUCTION}

$\mathbf{C}$ OUPLED resonator optical waveguides (CROWs) [1]-[10] are composed of a periodic array of isolated structural elements (e.g., high-Q resonators such as defects in two-dimensional (2-D) photonic crystal slabs [11]-[13] — see Fig. 1) weakly coupled to one another [14]. Waveguiding is fundamentally a consequence not of total internal reflection or Bragg reflection from a periodic structure, but instead of the overlap between the resonator modes of the structural elements, although the individual resonator modes can depend on Bragg reflection. In direct correspondence with the description of electrons in a strong periodic potential in solid state physics [15], [16], the waveguide modes of a CROW can be described using the tight binding approximation (Section II). Light propagates in such a waveguide, based on nearest neighbor coupling, following the physical layout of the resonators-a "photon hopping" model, thereby allowing the manipulation of the light path on a microscopic scale.

CROWs are not limited to coupled defects in photonic crystals and can be realized in the coherent coupling of whisperinggallery modes in microspheres [17] or in optical fibers [18], as will be discussed in Section V. In this paper, we will focus mainly on CROWs as planar waveguides in photonic crystals, as some of the remarkable properties of such waveguides listed below make them particularly appealing from a designer's perspective and for applications:

1) The extensive research on the properties of defects in photonic crystals [11], [19], [20] directly leads to both analytical [2], [5], [9] and numerical [2], [21] descriptions of the waveguide modes and the propagation of pulses.

Manuscript received December 13, 2001; revised February 19, 2002. This work was supported by the US Office of Naval Research and by the US Air Force Office of Scientific Research.

The authors are with the Departments of Electrical Engineering and Applied Physics, 128-95 California Institute of Technology, Pasadena, CA 91125 USA (e-mail: shayan@caltech.edu).

Publisher Item Identifier S 1077-260X(02)05468-0.

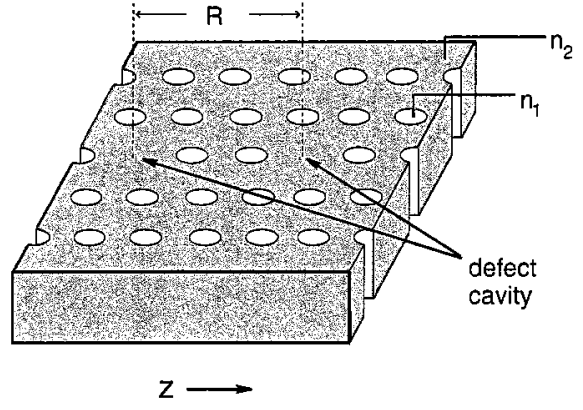

Fig. 1. Schematic of an infinitely-long 1-D CROW with periodicity $R$ consisting of defect cavities embedded in a 2-D photonic crystal.

2) The group velocity in CROWs can be several orders of magnitude smaller than in bulk material (of the same refractive index) [1]. This leads to an important class of applications [8], such as photorefractive holography for all-optical buffers in packet-switched optical networks [6], highly efficient second harmonic generation [7] etc.

3) CROWs can be defined as a single (or a few) waveguiding band(s) inside the photonic bandgap with the guided mode(s) well isolated from the continuum of modes that lie outside the bandgap. This is in contrast with band-edge waveguides in photonic crystals, which can also achieve low group velocity, but usually at the cost of poor confinement of the field to the desired modes.

In the applications of CROWs as information processing devices [e.g., second-harmonic generation, correlators, four-wave mixing (FWM), photorefractive holography], the study of pulse propagation is important as the case of practical importance, and it involves features not evident in a discussion limited to continuous-wave $(\mathrm{CW})$ phenomena. Section III discusses the propagation of pulses both in the linear dispersion approximation and involving the higher order dispersion terms to all orders.

The fundamental representation of pulse propagation in a CROW that represents the effects of a nonlinear electric polarization generated by optical fields is discussed in Section IV. In particular, the use of a modulated spatio-temporal Bloch wavefunction is the starting point for the analysis of all nonlinear optical phenomena in the coupled mode framework for this family of waveguides. Examples of the application of this analysis include second-harmonic generation (Section IV-A), and photorefractive holography in the framework of FWM [6].

This paper discusses how the analysis of nonlinear phenomena in a new family of waveguides can be built ab initio from a study of the basic waveguiding physics, investigating first the effects of dispersion, and then the nonlinear polarization. The insights gained by the analysis of CROWs are applicable to other forms of photonic crystal waveguides. With growing emphasis on the design of waveguides in photonic crystals for practical applications, CROWs stand out in the light of a well-understood analytical characterization that highlights 
the role of the structural parameters in waveguiding, accompanied by detailed numerical simulations and experimental corroboration of the theory.

\section{CROW WAVEGUIDE MODES}

We assume that the structural elements comprising the periodic waveguide of length $L$, e.g., defects in a 2-D photonic crystal slab with index confinement in the out-of-plane direction, are identical and lie along the $z$ axis separated by a distance $R$. The waveguide mode (an eigenmode of a time-independent Hamiltonian) $\phi_{k}(z)$ at a particular propagation constant $k$ is written as a linear combination of the individual eigenmodes $\psi_{l}(\mathbf{r})$ of the elements that comprise the structure [15]

$$
\phi_{k}(\mathbf{r})=\sum_{n} \exp (-i n R \mathbf{k} \cdot \hat{z}) \sum_{l} \psi_{l}(\mathbf{r}-n R \hat{z})
$$

where the summation over $n$ runs over the $N=L / R$ structural elements and the summation over $l$ refers to the bound states in each individual element. In a photonic crystal CROW, for instance, the individual resonator modes are doubly degenerate [2], so that $l= \pm 1$.

In the description of a one-dimensional (1-D) waveguide of finite length, the mode propagation constant $k=|\mathbf{k}|$ is restricted according to the Born-von Karman periodic boundary condition [15]

$$
k_{m}=m\left(\frac{2 \pi}{L}\right), \quad m=0,1,2, \ldots
$$

where $m$ is an integer; $k_{m}$ then ranges over the Brillouin zones and because $\phi_{k}(\mathbf{r})$ is of the Bloch form [15], we may only consider the first Brillouin zone $m=0,1, \ldots, N-1$ to characterize the dispersion relationship in the structure [1]. From (2), $\Delta k \equiv k_{m+1}-k_{m}=2 \pi / L$ so that in the description of long structures, the discrete distribution of eigenmodes goes over to a continuous spectrum.

For most applications, the emphasis is on undistorted pulse propagation and a characterization of the deviations from ideality. Consequently, we will assume that the structure is designed so that a single dispersion relationship describes the multiplicity of $l$ resonator modes in (1). If a single $l$ is insufficient to characterize the lowest order mode of an individual resonator, this approximation is still valid if the lowest order modes are degenerate in frequency, as is the case for lowest order modes that differ by parity [2]. (The two consequent waveguide modes have opposite parity and cannot couple to each other.)

The dispersion relationship for a CROW around a central wave number $k_{0}$ is

$$
\begin{aligned}
\omega_{k_{0}+K} & =\Omega(1-\Delta \alpha / 2)+\Omega \kappa \cos (K R) \\
& \equiv \omega_{0}+\Delta \omega \cos (K R)
\end{aligned}
$$

where $\Omega$ is the eigenfrequency of the individual resonators, and both $\Delta \alpha$ and $\kappa$ are overlap integrals involving the individual res- onator modes and the spatial variation of the dielectric constant [1]

$$
\begin{aligned}
\Delta \alpha= & \int d^{3} \mathbf{r}\left[\epsilon_{\mathrm{wg}}\left(\mathbf{r}-R \mathbf{e}_{\mathbf{z}}\right)-\epsilon_{\mathrm{res}}\left(\mathbf{r}-R \mathbf{e}_{\mathbf{z}}\right)\right]\left|\psi_{l}(\mathbf{r})\right|^{2} \\
\kappa= & \int d^{3} \mathbf{r}\left[\epsilon_{\mathrm{res}}\left(\mathbf{r}-R \mathbf{e}_{\mathbf{z})}-\epsilon_{\mathrm{wg}}\left(\mathbf{r}-R \mathbf{e}_{\mathbf{z}}\right)\right]\right. \\
& \cdot \boldsymbol{\psi}_{l}(\mathbf{r}) \cdot \psi_{l}\left(\mathbf{r}-R \mathbf{e}_{\mathbf{z}}\right)
\end{aligned}
$$

where $\epsilon_{\mathrm{res}}$ is the dielectric constant of the individual resonators, and $\epsilon_{\mathrm{wg}}$ is the dieletric constant of the waveguide.

The formation, when the number of resonators is increased, of a continuous dispersion relationship such as (5) from a discrete spectrum by reduction of the mode energy splitting of the individual resonators has been discussed elsewhere [22], [23]. In the limit of nearest-neighbor coupling, as applicable to waveguides formed by coupling high-Q resonators, the parameter $\Delta \omega$ in (3) is given in terms of the spatial variation of the dielectric constant by $\Delta \omega=\Omega \kappa$. For the 2-D photonic crystal structures, we discuss in this paper, finite-difference time-domain simulations indicate that $|\Delta \omega / \Omega| \sim 10^{-3}-10^{-4}[2]$, and similar numbers have been measured for CROWs constructed of polystyrene microspheres and microcavities in lithographically patterned in GaAs (see Section V).

If we assume that $\Omega \sim \omega_{0}$ in order of magnitude, then to a first approximation, $|\Delta \omega| \ll \omega_{0}$ and the dispersion relationship of the waveguide mode is approximately linear in the middle of the bandgap (the group velocity goes to zero at the band edges). We can write an approximate dispersion relationship around the central wave vector of the pulse $k_{0}$ as

$$
\omega\left(k_{0}+K\right)=\omega\left(k_{0}\right)+\left.\frac{d \omega}{d k}\right|_{k=k_{0}} K+\cdots \approx \omega_{0}+v_{g} K
$$

where $v_{g}$ is the group velocity of the pulse. Pulse propagation governed by (5) will be discussed in Section III-A, and as governed by the nonlinear relationship (3) in Section III-B.

The spatial distributions of the individual resonator wavefunctions decay exponentially away from the center of the defect structure [24], typically with a length scale on the order of the wavelength of light. The quality factors are limited, ideally, by the lifetime of the defect mode as a consequence of the dielectric loss in the material [11], and it may be shown by introducing the absorption coefficient $\sigma(\mathbf{r})$ into Maxwell's equations that [2]

$$
Q=\frac{\omega}{4 \pi}\left[\frac{\int d^{3} \mathbf{r} \epsilon(\mathbf{r}) \phi_{k}(\mathbf{r})^{*} \cdot \phi_{k}(\mathbf{r})}{\int d^{3} \mathbf{r} \sigma(\mathbf{r}) \phi_{k}(\mathbf{r})^{*} \cdot \phi_{k}(\mathbf{r})}\right]
$$

By altering the distribution of the refractive index, e.g., using the electro-optic effect, or changing the physical structure of the waveguide, e.g., using piezoelectric actuators, the waveguiding bandwidth $2 \Delta \omega$ and the group velocity $v_{g}$ can be manipulated. Since waveguiding in CROWs comprised of high-Q resonators is itself a consequence of the weak overlap between the tails of the eigenmodes [see (4)], a strong perturbation is not needed in order to produce an appreciable difference in the propagation behavior. 


\section{Dispersive Pulse Propagation}

In practical applications, waveguides are designed to realize undistorted propagation, coupling, harmonic generation, etc., over a certain length of propagation. It is, therefore, important to characterize how a given pulse, as the physical carrier of information, propagates with the role of the structural parameters $\left(R, v_{g}, \Delta \omega\right.$ etc.) clearly highlighted.

The field describing a pulse $\mathcal{E}(\mathrm{r}, t)$ is written as a superposition of waveguide modes $\phi_{k}(\mathbf{r})$ within the Brillouin zone with the corresponding time-evolution propagators (as appropriate for any linear and time-invariant system)

$$
\begin{aligned}
\mathcal{E}(\mathbf{r}, t) & \approx \int \frac{d k}{2 \pi} e^{i \omega_{k} t} c_{k} \phi_{k}(\mathbf{r}), \\
& =e^{i \omega_{0} t} \int_{-\pi / R}^{\pi / R} \frac{d K}{2 \pi} e^{i \Delta \omega t \cos (K R)} c_{k_{0}+K} \boldsymbol{\phi}_{k_{0}+K}(\mathbf{r}) .
\end{aligned}
$$

If the input field involves a wider range of $K \mathrm{~s}$, the limits of integration can be extended appropriately, but the analysis of Section III-B is then more relevant than that of Section III-A.

The boundary conditions that arise in pulse propagation problems typically specify a pulse shape at the $r=0$ cross section of the waveguide and centered at the optical frequency $\omega_{0}$

$$
\mathcal{E}(\mathbf{r}=0, t)=e^{i \omega_{0} t} E(z=0, t) \hat{\mathbf{u}}
$$

where $\hat{\mathbf{u}}$ is a unit magnitude vector that describes the vectorial nature of the field at $\mathbf{r}=0$.

In describing the propagation of a pulse, we seek a closed form expression for $\mathcal{E}(\mathrm{r}, t)$, given $\mathcal{E}(\mathrm{r}=0, t)$. It is useful to consider a simpler more specific case first.

\section{A. Linear Dispersion Approximation}

In the linear dispersion (dispersionless) approximation of (5), (7) can be written as

$$
\mathcal{E}(\mathbf{r}, t)=e^{i \omega_{0} t} \int_{-\pi / R}^{\pi / R} \frac{d K}{2 \pi} e^{i v_{g} t K} c_{k_{0}+K} \phi_{k_{0}+K}(\mathbf{r})
$$

We may extend the limits of integration to infinity by defining the coefficients (as functions of $K$ ) so that they are zero outside the first Brillouin zone. From the equality of (9) evaluated at $z=0$ and (8), it follows that the vectorial behavior of $\phi_{k_{0}+K}(0)$ must follow $\hat{\mathbf{u}}$. Further, by inverting the Fourier transform relationship, we write

$$
c_{k_{0}+K}=\frac{1}{\phi_{k_{0}+K}(0)} \int d\left(v_{g} t^{\prime}\right) E\left(z=0, t^{\prime}\right) e^{-i v_{g} t^{\prime} K}
$$

in terms of the scalar $\phi_{k_{0}+K}(0)=\left|\phi_{k_{0}+K}(0)\right|$. Combining (9) and (10)

$$
\begin{aligned}
& \mathcal{E}(\mathbf{r}, t)=e^{i \omega_{0} t} \int d\left(v_{g} t^{\prime}\right) E\left(z=0, t^{\prime}\right) \\
& \cdot \int \frac{d K}{2 \pi} \frac{\boldsymbol{\phi}_{k_{0}+K}(\mathbf{r})}{\phi_{k_{0}+K}(0)} e^{i v_{g}\left(t-t^{\prime}\right) K} .
\end{aligned}
$$

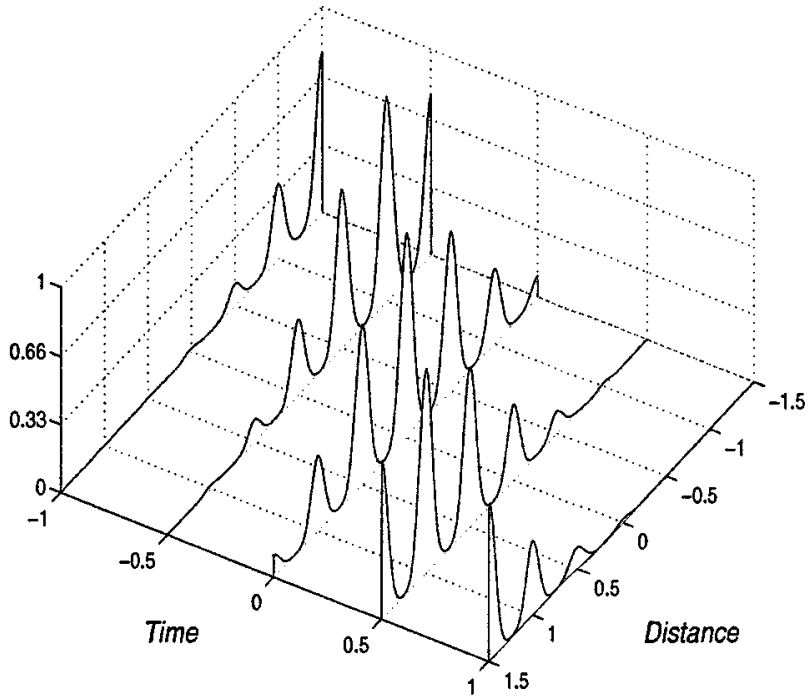

Fig. 2. Pulse propagation in a CROW in the dispersionless approximation, highlighting the spatial modulation of the envelope due to the physical structure of the waveguide. The "Distance" axis is normalized to $R$. Parameterized by time, the envelope is graphed as a function of (norm.) distance; the pulse moves toward positive values of $z$ as $t$ increases, i.e., from the back to the front in this representation.

As discussed in [5], the approximations

$$
\left[\phi_{k_{0}+K}(0)\right]^{-1} \approx 1-\sum_{l} \psi_{l}(R) 2 \cos \left[\left(k_{0}+K\right) R\right] \approx 1
$$

are usually well justified in CROWs comprised of high-Q resonators. A few algebraic manipulations (in the form of Fourier transform relationships [5]) show that

$$
\begin{aligned}
\mathcal{E}(\mathbf{r}, t)=e^{i \omega_{0} t} \sum_{n} e^{-i k_{0} n R} \sum_{l} b_{l} \psi_{l}(\mathbf{r}-n R \hat{z}) & \\
& \cdot \sum_{m} E\left(z=0, t-\frac{n R+m L}{v_{g}}\right)
\end{aligned}
$$

where the summation over $n=0,1, \ldots, N-1$ indexes the resonators that comprise the CROW, and the summation over $m$ reflects the Born-von Karman quantization of the propagation constant $k$ in structures of finite length (2). Fig. 2 shows the magnitude of the envelope of Gaussian pulse propagating along a CROW; the spatial distribution is dictated by the $\psi \mathrm{s}$. As mentioned before, the vectorial behavior of $\mathcal{E}(\mathbf{r}, t)$ is simply given by that of $\psi_{l}(\mathbf{r})$; therefore, we will usually work in the $(1+1) D$ approximation ( $z$ and $t$ coordinates), and revert back the $(3+1) D$ expressions when necessary, e.g., (33).

Since the allowed $k \mathrm{~s}$ are quantized in a structure of finite length, the dispersion relationship (5) implies that the allowed $\omega=\omega_{0}+\Omega$ values are quantized. To avoid aliasing [25], the temporal interval between two samples $2 \pi / \Delta \Omega$ must be greater than twice the temporal extent of the pulse envelope $T$, where $\Delta \Omega=v_{g} \Delta K$ according to (5). Therefore

$$
\frac{2 \pi}{(2 \pi / L) v_{g}}=2 T_{\max } \quad \text { which implies } \quad T_{\max }=\frac{1}{2} \frac{L}{v_{g}} .
$$

Further, Fig. 2 shows that the $z$ projection of the eigenmode $\phi_{k}(\mathrm{r})$ represents a spatial sampling function for the propa- 
gating pulse envelope, especially in the limit that the individual structural eigenmodes $\psi_{l}(\mathbf{r})$ are tightly confined (high-Q resonators). Again, to avoid aliasing, it is necessary that the spectral content in $K$ space be no greater than $K_{\max }$

$$
\frac{2 \pi}{R}=2 K_{\max } \quad \text { which implies } \quad K_{\max }=\frac{1}{2}\left(\frac{2 \pi}{R}\right) .
$$

But the free space pulse envelope is invariant in the frame $z-$ $v_{g} t$, and this maximum $K$ space extent translates into a minimum pulsewidth $T_{\min }$,

$$
\frac{1}{2} v_{g} T_{\min }=R \quad \text { which implies } \quad T_{\min }=\frac{2 R}{v_{g}} .
$$

In a structure of finite length described by the tight-binding approximation, therefore, there exists both a maximum and a minimum allowed pulse duration; the former arises because of the finite length of the structure, and the latter because of the sampling-train-like eigenmodes of the waveguide. Equation (16) limits the bandwidth (where most of the energy of the pulse is concentrated) in the Fourier $K$ space associated with the propagation distance $z$, and since the pulse is propagating with group velocity $v_{g}$, also in the Fourier $\Omega$ space associated with the temporal coordinate $t$. The dimension of the space of finite-energy signals (pulse envelopes) that are identically zero outside the time interval $\left[-T_{0} / 2, T_{0} / 2\right]$ and have most of their energy concentrated in the bandwidth $\left[-\Omega_{0} / 2, \Omega_{0} / 2\right]$ is approximately $D=\Omega_{0} T_{0}+1$ [26]

$$
D=\left(\frac{2 \pi}{R} v_{g}\right)\left(\frac{1}{2} \frac{L}{v_{g}}\right)+1=\pi N+1
$$

where $N=L / R$ is the number of resonators in the waveguide. These pulse envelopes can be represented in the mean-square sense by a superposition of the prolate spheroidal wave functions within the interval $\left[-T_{0} / 2, T_{0} / 2\right] . D$ also gives a measure of the information theoretic capacity of a CROW as measured in bits using the simple ON-OFF keyed (OOK) amplitude modulation format.

\section{B. Higher Orders of Dispersion}

In this case, the coefficients $c_{k_{0}+K}$ are derived from the equality of (7) evaluated at $z=0$ and (8) in the scalar $(1+1) D$ notation

$$
E(z=0, t)=\int_{-\pi / R}^{\pi / R} \frac{d K}{2 \pi} e^{i \Delta \omega t \cos (K R)} c_{k_{0}+K} \phi_{k_{0}+K}(0) .
$$

The nonlinear term in the exponent implies that this is not a simple Fourier transform relationship. In considering higher order dispersion terms in the Taylor series expansion of the dispersion relationship, the integral equation (18) cannot in general be inverted to obtain the cs in closed form. This is clearly evident when, for example, the exponent involves terms of quadratic or higher polynomial powers of $K$. Therefore, rather than work with the successive terms in a Taylor-series expansion of the dispersion relationship, we will maintain the full form of (3).
Note that the dispersion relationship (3) is symmetric about $K=0$. We will assume that $E(z=0, t)$ is a symmetric envelope, but this is merely for analytical convenience. Consequently, $c_{k_{0}+K} \phi_{k_{0}+K}(0)=c_{k_{0}-K} \phi_{k_{0}-K}(0)$ for all $K$ within the first Brillouin zone.

A change of variables highlights the mathematical structure of (18)

$$
\begin{aligned}
\varphi & \equiv K R \\
x & \equiv \Delta \omega t \\
h(\phi) & \equiv c_{k_{0}+K} \phi_{k_{0}+K}(0) \\
f(x) & \equiv 2 R E(z=0, x / \Delta \omega)
\end{aligned}
$$

so that (18) becomes

$$
\pi f(x)=\int_{-\pi}^{\pi} d \varphi e^{i x \cos \varphi} h(\varphi)
$$

where $f(x)$ is a known function, in terms of which we want to find $h(\varphi)$. For almost all cases of practical interest, we can instead find the coefficients in the expansion of $h(\varphi)$ as a Fourier cosine series

$$
h(\varphi)=\sum_{n=0}^{\infty} c_{n} \cos (n \varphi) .
$$

Using the identity [27, (9.4-5)]

$$
e^{i x \cos \varphi}=\sum_{m=0}^{\infty} b_{m} J_{m}(x) \cos (m \varphi),
$$

where

$$
b_{m}= \begin{cases}1, & m=0 \\ 2 i^{m}, & m \geq 1\end{cases}
$$

and the orthogonality of the cosines over the interval $(-\pi, \pi)$, we can simplify (20) to

$$
f(x)=\sum_{n=0}^{\infty}\left(b_{n} c_{n}\right) J_{n}(x) \equiv \sum_{n=0}^{\infty} a_{n} J_{n}(x) .
$$

Therefore, if we can expand $f(x)$ [which describes the envelope at the $z=0$ cross section-see (19)] in a Neumann series of Bessel functions [28, Ch. IX], we can find the coefficients $c_{n}$, and by subsequently using (21) and (19), the coefficients $c_{k_{0}} \pm K$.

The general approach to finding the $a_{n} \mathrm{~s}$ is based on a contour integral involving the Neumann polynomials, but alternative approaches are useful in yielding approximate but simpler representations. Defining the coefficients

$$
\beta_{n}= \begin{cases}\frac{1}{2 R} c_{k_{0}}, & n=0 \\ \frac{n}{i^{n}} \int_{0}^{\infty} \frac{d t^{\prime}}{t^{\prime}}\left[E\left(0, t^{\prime}\right)-E(0,0)\right] J_{n}\left(\Delta \omega t^{\prime}\right) & n \geq 1\end{cases}
$$

where $c_{k_{0}}$ is a constant that arises from Parseval's relationship, leads to the representation [9]

$$
\begin{array}{r}
\mathcal{E}(\mathbf{r}, t)=\frac{1}{4} e^{i \omega_{0} t} \sum_{m=0}^{\infty} b_{m} J_{m}(\Delta \omega t) \sum_{n=0}^{\infty} \beta_{n} G\left(\mathbf{r}_{\perp}\right) \\
\cdot\left\{e^{-i( \pm m \pm n)_{+} k_{0} R} \sum_{l} \psi_{l}\left[\mathbf{r}-( \pm m \pm n)_{+} R \mathbf{e}_{\mathbf{z}}\right]\right\}
\end{array}
$$




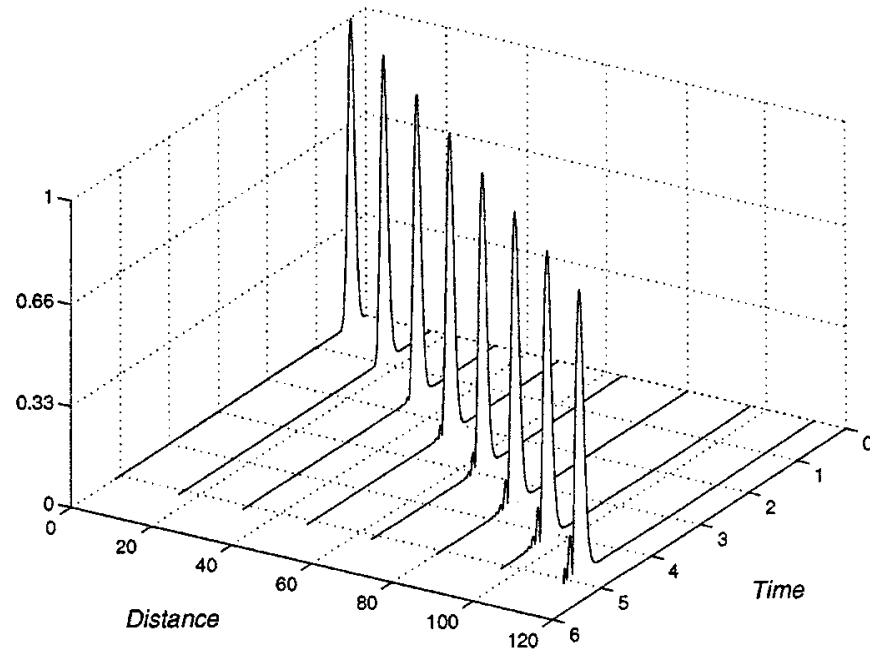

Fig. 3. Temporal evolution of a Gaussian envelope at specific distances inside a CROW, showing the effects of dispersive propagation. Distance is normalized to units of $R$, and the time scale describes a Gaussian envelope at $z=0$. At greater depths, the peak of the envelope arrives at a later time, and ripples in the trailing edge indicate higher order distortion.

where $G\left(\mathbf{r}_{\perp}\right)$ describes the transverse mode profile. We have used the symbol " ()$_{+}$" in (25) as a compact notation for the sum over both choices of sign of " \pm " that yield a nonnegative number for the expression inside the brackets.

Equation (25) describes the propagation of an arbitrary pulse envelope $E(z=0, t)$ in the CROW waveguide to all orders of dispersion. Intuitively, the Bessel functions in this nonlinear problem assume the role of sinusoids as basis functions in the linear dispersion approximation [10]. Equation (25) may be separated into two terms describing the forward and backward propagating envelopes. A pulse envelope, as a function of $t$, is described by a contiguous set of Bessel functions: the field at $z$ is written as a superposition of an appropriately translated set of these functions $\left\{J_{z / R}(t), J_{z / R+1}(t), \ldots\right\}$, multiplied by the coefficients $\left\{\beta_{0}, \beta_{1}, \ldots\right\}$. Distortion accumulates with distance as a consequence of the changing inter-relations between neighboring Bessel functions, e.g., the difference between $\left[\beta_{0} J_{0}(t)+\beta_{1} J_{1}(t)+\cdots+\beta_{p} J_{p}(t)\right]$ and $\left[\beta_{0} J_{5}(t)+\beta_{1} J_{6}(t)+\cdots+\beta_{p} J_{5+p}(t)\right]$.

Temporal profiles of a Gaussian pulse at increasing depths into the CROW are depicted in Fig. 3 and clearly show the effects of accumulated dispersion. While it is evident that the peak of the envelope reaches structural elements farther from the input at a later time $\Delta z / \Delta t \sim \Delta \omega R$, there is no single parameter that describes propagation governed by the nonlinear dispersion relationship to arbitrary distances. Equation (25) is, nevertheless, in a simple form for numerical evaluation for a particular case, e.g., the Gaussian envelope in Fig. 3. Further details of the dependence of the propagating envelope on the design parameters of the CROW [e.g., $\Delta \omega$ appearing in (3)] are discussed in detail elsewhere [9], [10].

\section{COUPled-Mode Theory of Pulses}

Many of the nonlinear phenomena in CROWs involving pulses arise from a nonlinear polarization generated by the fields in the waveguide. Coupled-mode theory [27] dictates how this polarization drives the evolution of the amplitude (envelope) of the waveguide modes, typically under the slowly varying envelope approximation. As we have seen in Section II, the waveguide modes of a CROW are more complicated than plane waves; furthermore a pulse comprises a number of CROW waveguide modes [as in (7)] and each component can acquire its own envelope function, which complicates the analysis even more. We show that in most practical cases, the formalism can be concisely expressed using a spatio-temporal Bloch wavefunction. The approximations involved in this derivation, which translate into bounds on the structural parameters of the CROW, are most easily understood in the spectral (Fourier transformed) domain. Since we are interested in undistorted signal propagation and processing applications, we will assume that the conditions of Section III-A apply and work in the dispersionless approximation.

A change in the power carried by the modes due to a driving polarization is described by including a $z$ dependency in the coefficients $b_{k_{0}+K}$. These coefficients will account for not only the effect of the polarization, but also the initial superposition of $k$ s that comprise the pulse at e.g., the $z=0$ reference "edge" of the waveguide. It is convenient to separate these two components of $b_{k_{0}+K}(z)$ and write $b_{k_{0}+K}(z) \rightarrow c_{k_{0}+K} \tilde{A}_{k}(z)$, where $c_{k_{0}+K}$ is independent of $z$ and $\tilde{A}_{k}(z=0)=1$. Thus

$$
\mathcal{E}(z, t)=e^{i \omega_{0} t} \int \frac{d K}{2 \pi} c_{k_{0}+K} \tilde{A}_{k_{0}+K}(z) e^{i v_{g} t K} \phi_{k_{0}+K}(z) .
$$

As in (10), the coefficients $c_{k_{0}+K}$ arise from the boundary condition, and an expression similar to (13) is obtained

$$
\begin{aligned}
\mathcal{E}(z, t)= & e^{i \omega_{0} t} \sum_{n} e^{-i k_{0} n R} \sum_{l} \psi_{l}(z-n R)\left[\int d\left(\left|v_{g}\right| t^{\prime}\right)\right. \\
& \left.\cdot E\left(z=0, t^{\prime}\right) A\left(z ; v_{g}\left[t-\frac{n R}{v_{g}}\right]-v_{g} t^{\prime}\right)\right]
\end{aligned}
$$

where the envelope $A$ depends on two spatial coordinates, $z$ and the "auxiliary" spatial coordinate $\zeta \equiv v_{g}\left(t-n R / v_{g}\right.$ ) (as a convolution integral in the latter variable).

Taking the temporal Fourier transform of (27), e.g., with definitions $\tilde{\mathcal{E}}(z, \Omega)=\int d t \exp (i \Omega t) \mathcal{E}(z, t)$ and similarly for $E$ and $A$, it may be shown after some algebra that (8)

$$
\begin{aligned}
\tilde{\mathcal{E}}(z, \Omega)=\sum_{n} e^{i\left(\kappa-k_{0}\right) n R} \sum_{l} \psi_{l}(z-n R) & \\
& \times \tilde{E}\left(z=0, \omega_{0}+\Omega\right) \tilde{A}\left[z ; \frac{\omega_{0}+\Omega}{v_{g}}\right] .
\end{aligned}
$$

The group velocity $v_{g}$ plays an important role in (28): as $v_{g}$ is reduced, the contributing spectral components of the envelope $\tilde{A}$ are at frequencies farther removed from dc, i.e., for fixed $\Omega$, the argument of $\tilde{A}$ is larger in magnitude, signifying that an envelope of broader bandwidth is described. If CROWs are to be designed for a substantial (at least three to four orders of magnitude), reduction in the group velocity compared to bulk material waveguides [1], it follows that $\tilde{A}$ needs to be broadband in the time-transformed Fourier space. As we have not affected the $z$ part of $A$, we approximate $A(z, \zeta-Z) \rightarrow \alpha(z) \delta(\zeta-Z)$ using the delta function in its usual role in (28). (This follows from the 
fact that the inverse Fourier transform of a broad-band function in Fourier space is a narrowband waveform in original space, which in this case refers to the auxiliary spatial coordinate.)

A different but related physical argument leads to the same conclusion: in the slowly varying envelope approximation, the polarization term driving the evolution of the field may be assumed to be constant over the relatively small range of propagation constants $k_{0} \pm K$ that comprise a narrow-band pulse in the center of the waveguide band.

In this approximation, (27) simplifies to

$$
\mathcal{E}(z, t)=\alpha(z) e^{i\left(\omega_{0} t-k_{0} z\right)} u_{k_{0}}(z, t)
$$

where we define

$$
\begin{aligned}
& u_{k(\omega)}(z, t)=\sum_{n} e^{i k_{\omega}(z-n R)} \sum_{l} \psi_{l}(z-n R) \\
& \times E\left(z=0, t-\frac{n R}{v_{g}}\right)
\end{aligned}
$$

and the notation $k_{\omega} \equiv k(\omega)$ is a reminder of the presence of a dispersion relationship as in (5).

In considering CROWs comprising a large number of structural elements, the summation over $n$ in (30) may be translated as desired, and $\exp \left[i\left(\omega_{0} t-k_{0} z\right)\right] u_{k_{0}}(z, t)$ is of the Bloch form (in spatial and temporal coordinates), i.e., a plane wave multiplying a function with an inherent periodicity reflecting that of a lattice structure. Consequently, $\mathcal{E}(z, t)$ may be viewed as an amplitude $\alpha(z)$ modulating the Bloch wavefunction $u_{k_{0}}(z, t)$. The spatial periodicity of the Bloch wavefunction is exactly that of the CROW structure and the temporal periodicity is the time taken by the pulse envelope to travel the distance defining the spatial periodicity.

The generalization of the spatial dependency of the waveguide modes while maintaining the assumption of a single spatial dimension for pulse propagation, is straightforward since the only the vectorial functions in (1) are the individual resonator modes. The Bloch wave function is normalized according to an inner product definition (in three spatial and one temporal coordinates) between the vector space of the Bloch wave function and its dual space

$$
\int \frac{d t}{T} \int d \mathbf{r} \epsilon(\mathbf{r})\left[\mathbf{u}_{k(\omega)}(\mathbf{r}, t)\right]^{*} \cdot \mathbf{u}_{k(\omega)}(\mathbf{r}, t)=1
$$

where the spatial integration extends over a unit cell and the temporal integration over the extent of the pulse envelope, with a characteristic time constant $T$. The temporal normalization is needed to ensure that (31) represents an electromagnetic energy conservation relationship, and can be interpreted as yielding a time-averaged energy stored in a unit cell volume. Note that the involvement of $T$ is indicative of the nontrivial role that pulse envelopes play in propagation phenomena in CROWs, as discussed in Section III-A.

The starting point for the analysis of nonlinear phenomena such as second-harmonic generation (Section IV-A) and FWM [6] is to write the field under consideration (such as the secondharmonic field) in the modulated spatio-temporal Bloch representation (29), and obtain equations of motion for the envelope of that representation.

\section{A. Second-Harmonic Generation}

Following (29), the Ansatz describing a pulse at the second harmonic (carrier) frequency $2 \omega$ is

$$
\mathbf{E}_{2}(\mathbf{r}, t)=E_{2}(z) e^{i 2 \omega t} e^{-i k_{2 \omega} z} \mathbf{u}_{k(2 \omega)}(\mathbf{r}, t)
$$

and is generated by a nonlinear polarization $\mathbf{P}_{\mathrm{NL}}(\mathbf{r}, t)$ according to

$$
\nabla \times\left[\nabla \times \mathbf{E}_{2}\right]+\frac{\epsilon(\mathbf{r})}{c^{2}} \frac{\partial^{2} \mathbf{E}_{2}}{\partial t^{2}}=-\frac{1}{c^{2}} \frac{\partial^{2}}{\partial t^{2}} \mathbf{P}_{\mathrm{NL}}(\mathbf{r}, t) .
$$

Each term of the above equation can be expanded out in terms of the components of (32) and using the slowly varying approximation [7]. To show the correspondence with second-harmonic generation in bulk materials, we will only discuss a particular case of the final result here. We assume that the field at the fundamental, described by the propagation constant $k_{1}(\omega)$ is undepleted, and define the propagation constant mismatch

$$
\Delta k_{n} \equiv 2 k_{1}(\omega)-k(2 \omega)+n \frac{2 \pi}{R}
$$

which explicitly involves the Bragg contribution of the periodic waveguide. The second-harmonic field in the unsaturated limit grows according to

$$
\begin{aligned}
E_{2}^{(0)}(z)=-i & {\left[\frac{\sin \left[\Delta k_{n} z / 2\right]}{\Delta k_{n} z / 2}\right] e^{-i \Delta k_{n} z / 2} } \\
& \times z E_{1}^{2} \frac{\omega}{v_{2}}\left[D_{n}^{(0)}-i \frac{1}{\omega} D_{n}^{(1)}-\frac{1}{4 \omega^{2}} D_{n}^{(2)}\right]
\end{aligned}
$$

where the $D$ s are complicated space-time integrals involving the overlap of adjacent resonator eigenmodes and the effective second-order nonlinearity coefficient of the medium, and $v_{2}$ is the group velocity at the second-harmonic frequency [7].

Setting aside the modifications brought about by the $D$ coefficients, the (quasi) phase-matching sinc function in (35) is exactly analogous to the results of $\mathrm{CW}$ second-harmonic generation in bulk crystals. Equation (35) also shows that at the phase-matched condition, the intensity of the second harmonic $\left|E_{2}\right|^{2}$ grows quadratically with distance $z$, the intensity of the fundamental $\left|E_{1}\right|^{2}$, and in regions where it is a constant, the nonlinearity coefficient $\tilde{d}$ (through the $D$ s); these are features in common with the analysis of second-harmonic generation in bulk media [29]. Further discussions of the more general analysis for second-harmonic generation, including a discussion of the Hellman-Feynman theorem in the description of the group velocity, and a measure of the efficiency of second-harmonic generation, are discussed elsewhere [7].

\section{B. Two-Wave and Four-Wave Coupling via Index Gratings}

The interference pattern of two co-incident pulses in a nonlinear CROW creates an index grating that may cause power transfer from one waveform to another-this is known as two-beam coupling. Phenomena such as FWM and holography involve a third waveform which is Bragg scattered from the grating, resulting in the generation of a fourth field that is related to the waveforms of the other fields. CROWs are an attractive geometry to realize the proposals of all-optical temporal signal processing using "nonlinear delay lines" [30]. 
From (13), the spatial Fourier transform (in $K$ space) of $\mathcal{E}(z, t)$ may be written as

$$
\begin{array}{r}
\tilde{\mathcal{E}}(K, t) \equiv \int \mathcal{E}(z, t) e^{-i K z} d z=e^{i \omega_{0} t} \sum_{n} e^{-i\left(k_{0}+K\right) n R} \\
\times \tilde{\psi}(K) E\left(z=0, t-\frac{n R}{v_{g}}\right) .
\end{array}
$$

A typical geometry involves a grating written by two counterpropagating pulses. If two pulses centered at the carrier frequencies $\omega_{1}$ and $\omega_{2}$, propagate in opposite directions with wave vectors $k_{1}$ and $-k_{2}$, and group velocities $v_{1}$ and $v_{2}$, respectively, the $K$ space representation of the field is

$$
\begin{aligned}
\tilde{\mathcal{E}}(K, t) & \\
= & e^{i \omega_{1} t} \sum_{n} e^{-i\left(k_{1}+K\right) n R} \tilde{\psi}(K) E_{1}\left(z=0, t-\frac{n R}{v_{1}}\right) \\
& +e^{i \omega_{2} t} \sum_{m} e^{-i\left(-k_{2}+K\right) m R} \tilde{\psi}(K) E_{2} \\
& \cdot\left(z=L, t-\frac{m R}{v_{2}}\right) .
\end{aligned}
$$

The interference pattern of the spectral components of this field (for example, considering the component at $K_{1}$ from the forward-propagating pulse and the component at $K_{2}$ of the backward-propagating pulse) is weighted by a complex coupling coefficient $\delta n\left(K_{1}, K_{2}\right)$ which depends on the material properties, the orientation of the medium, and the polarization of the waves [27], [31]. Therefore, we may write the grating as

$$
\begin{aligned}
\delta n(z, t)= & \frac{1}{F_{0}} e^{i\left(\omega_{1}-\omega_{2}\right) t} \sum_{n, m} \int \frac{d K_{1}}{2 \pi} \frac{d K_{2}}{2 \pi} e^{-i\left(K_{1}-K_{2}\right) z} \\
& \times \delta \hat{n}\left(K_{1}, K_{2}\right) e^{-i\left(k_{1}+K_{1}\right) n R} e^{-i\left(-k_{2}+K_{2}\right) m R} \\
& \times E_{1}\left(z=0, t-\frac{n R}{v_{1}}\right) E_{2}^{*}\left(z=L, t-\frac{m R}{v_{1}}\right) \\
& \times \tilde{\psi}\left(K_{1}\right) \tilde{\psi}^{*}\left(K_{2}\right)+\text { c.c. }
\end{aligned}
$$

where $F_{0}$ is the total optical power.

Equation (38), describing the refractive index, may be used to express the nonlinear polarization as in standard coupled-mode theory [27]; the formalism presented in Section IV then dictates the polarization-driven evolution of the fields. The particular example of photorefractive holography, which introduces the further complication of two time coordinates, separated by the hold-time of the grating is discussed elsewhere [6].

\section{EXPERIMENTAL PROGRESS AND APPLICATIONS OF CROWs}

The formalism we have described is independent of the material in which the CROW geometry is realized. In this section, we will review the progress made in the last few years in the fabrication of CROWs and related structures, and discuss their role in the demonstration of the theoretical validity of the framework in which our analysis was carried out. The particular realizations include: superstructure Bragg gratings in semiconductor materials and in fibers, polystyrene microspheres, alumina rods (microwave), resonators in GaAs without a photonic crystal structure, and defects in GaAs 2-D photonic crystal slabs. We also discuss three aspects of current research in this field that have close links to other areas of optics: holography and the photorefractive effect, spatially localized fields due to nonlinearity, and the coupling between waveguides that are intrinsically of different types.

The optical modes of a linear array of GaAs cavities with InGaAs quantum wells as the optically active material, were measured by angle-resolved photoluminescence spectroscopy, demonstrating the creation of a continuum of mode energy distributions from a discrete family as the number of resonators increases [23]. Narrowing the width of the GaAs channels connecting the cavities resulted in a larger bandgap at the Brillouin zone boundaries, in accordance with the increased modulation of the dielectric function along the waveguide.

Coupling between whispering gallery modes of two polystyrene microspheres was demonstrated to follow the tight-binding theory [17]. An intersphere coupling parameter [indicated by $\kappa$ in (4)] between microspheres of diameter 2 to $5 \mu \mathrm{m}$ was measured to be in the range $2.8-3.5 \times 10^{-3}$, and decreases as the size of the spheres increases since the field is less confined in smaller structures.

The validity of the tight-binding description of weakly coupled electromagnetic structures was demonstrated in the microwave $(10-13 \mathrm{GHz})$ experiments conducted in structures defined by micromachined alumina rods; a single rod was removed from consecutive unit cells, thereby forming a CROW; excellent agreement between the calculated and measured dispersion relationship of the resulting waveguiding band was observed (see [32, Fig. 4]).

The dispersion characteristics of a row of nine hexagonal cavities in a GaAs-based 2-D photonic crystal slab of length $8 \mu \mathrm{m}$ have been measured in the near IR [33]. The large defect cavities in this structure support several resonant modes [and would be indexed by $l$ in (1)]. Several transmission and stop bands ("minibands separated by minigaps") were observed within the photonic bandgap. Larger defect cavities were found to make it easier to couple light into and out of CROW waveguides, without necessarily invalidating the tight-binding model.

Superstructure gratings, [(SSGs), or superstructure Bragg gratings, (SBGs)] are gratings in semiconductor materials or in optical fibers, whose parameters in the description of the (periodic) refractive index variation themselves vary periodically along the length of the grating [18], [34], [35]. Typically, the SSG period is much longer than the period of the underlying uniform grating (the latter is on the scale of the wavelength of light). SSGs in semiconductor have been used in tunable DFB lasers, and in optical fibers for dispersion compensation of WDM systems [34]. A deep SSG is described by the tight-binding method [18]: in the weakly coupled regime, the eigenfield (waveguide mode) of an SSG is written as a superposition of the eigenmodes of the individual wells, exactly as in the description of a CROW.

Single pulse holographic recording requires strong nonlinear properties which are not available with ordinary materials since 
the hologram has only a short time and a limited amount of energy in which to form. The highly nonlinear properties of ultracold atomic vapors form the basis of one approach which was demonstrated recently [36]. We have investigated the possibility of recording and reconstructing holograms of optical pulses using photorefractive holography [37] in CROWs [6]. A CROW of weakly coupled high-Q resonators leads to very high optical intensities even at moderate (propagating) power levels - exactly what is required for holographic recording. In spite of the discrete localization of the optical field at the individual resonators, it is still possible to reconstruct faithfully the signal pulse which is recorded in the hologram. For photorefractive holography in photonic crystal CROWs, we refer to the schematic of Fig. 1: the material of index $n_{2}$ is taken to be photorefractive (e.g., GaAs) and the material of index $n_{1}$ will be assumed to be air for simplicity. Note that the defect cavity is then composed of photorefractive material, and the simultaneous presence of two optical fields in this region will induce a photorefractive index grating which can be used for holography.

A related family of waveguides in photonic crystals - the reduced-index and increased-index waveguides-is exemplified e.g., in a triangular lattice of holes by altering one row of holes [38], and either increasing or decreasing the radius [39] or leaving out a line of holes [40]. Photonic crystal waveguides fabricated in a silicon slab (with an undercut air region for symmetry) for both triangular and square lattice have demonstrated low scattering-loss transmission around bends at $1550 \mathrm{~nm}$ [41]. In light of the finite height of waveguides defined in photonic crystal slabs, (and the combination of photonic band guiding and index guiding that characterizes such waveguides), full vectorial calculations are important in the numerical determination of the waveguide modes of such structures, as in the analysis of CROWs [2]. One approach to the problem of coupling between CROWs and these types of waveguides is based on the design of adiabatically tapered transition regions [3], [4].

CROWs achieve a localization of the light field along the longitudinal direction of propagation, and the extension to 2-D or three-dimensional (3-D) CROW structures is straightforward conceptually. Localization of light in the transverse directions can be achieved in a variety of other ways. Index guiding in slab waveguides is the simplest of examples. In the presence of a nonlinear effect, intrinsic localized modes or discrete breathers can be formed [42]. This has been proven mathematically for using a class of time-periodic spatially localized solutions to a Hamiltonian coupled-oscillator nonlinear lattice [43]. Discrete breathers have been predicted for 2-D and 3-D photonic crystals with Kerr (cubic) nonlinearity [44], at nonlinear interfaces with quadratic nonlinearity [45] and along dielectric waveguide structures with a nonlinear Kerr-type response [46]. Unlike a CROW, the waveguides that have been under consideration, thus far, are uniform in the longitudinal direction. In the transverse plane, these waveguides may consist of an array of thin quadratically nonlinear layers embedded in a linear slab waveguide. Such structures can inhibit light propagation along the transverse axis under certain conditions, e.g., as a consequence of parametric coupling of the fundamental and second-harmonic fields excited at the nonlinear interfaces [47].

\section{CONCLUSION}

A CROW is a waveguiding structure based on the coupling of resonator modes that can be designed as planar waveguides e.g., in photonic crystals or as superstructure gratings in optical fibers but, in general, the analysis addresses any waveguide based on the spatial overlap of the wavefunctions defining the resonator modes. We have carried out a numerical and analytical characterization of such a waveguide, i.e., of the propagation of electromagnetic $\mathrm{CW}$ waves and pulses, and the first experimental realizations of CROWs corroborate the theory. We have also developed a spatio-temporal theory of nonlinear phenomena such as harmonic generation and holography in CROWs. In light of the applicability of the CROW concept to different physical manifestations of the coupling of resonators, and the degree to which the analytical characterization highlights, the role played in waveguiding by the structural parameters, we are optimistic that CROWs will play a significant role in the development of microstructure optical information processing devices.

\section{ACKNOWLEDGMENT}

The authors thank D. S. Cohen, A. Eyal, R. K. Lee, Y. Li, A. Scherer, and Y. Xu of the California Institute of Technology for useful discussions.

\section{REFERENCES}

[1] A. Yariv, Y. Xu, R. K. Lee, and A. Scherer, "Coupled-resonator optical waveguide: A proposal and analysis," Opt. Lett., vol. 24, pp. 711-713, Nov. 1999.

[2] Y. Xu, R. K. Lee, and A. Yariv, "Propagation and second-harmonic generation of electromagnetic waves in a coupled-resonator optical waveguide," J. Opt. Soc. Amer. B, vol. 17, no. 3, pp. 387-400, 2000.

[3] - "Adiabatic coupling between conventional dielectric waveguides and waveguides with discrete translational symmetry," Opt. Lett., vol. 25, no. 10, pp. 755-757, 2000.

[4] Y. Xu, Y. Li, R. K. Lee, and A. Yariv, "Scattering-theory analysis of waveguide-resonator coupling," Phys. Rev. E, vol. 62, no. 5, pp. 7389-7404, 2000.

[5] S. Mookherjea and A. Yariv, "Optical pulse propagation in the tightbinding approximation," Opt. Exp., vol. 9, no. 2, pp. 91-96, 2001.

[6] _ , "Optical pulse propagation and holographic storage in a coupledresonator optical waveguide," Phys. Rev. E, vol. 64, 2001.

[7] — "Second harmonic generation with pulses in a coupled-resonator optical waveguide," Phys. Rev. E, vol. 65, 2002.

[8] — "Characterization of polarization-driven pulse propagation in a coupled resonator optical waveguide," Opt. Lett., Nov. 2001.

[9] — , "Pulse propagation in a coupled resonator optical waveguide to all orders of dispersion," Phys. Rev. E, Mar. 2002, to be published.

[10] - "Nonlinear dispersion in a coupled-resonator optical waveguide," Opt. Lett., vol. 27, no. 11, pp. 933-935, 2002, submitted for publication.

[11] K. Sakoda, Optical Properties of Photonic Crystals. New York: Springer Verlag, 2001.

[12] J. D. Joannopoulos, R. D. Meade, and J. N. Winn, Photonic Crystals. Princeton, NJ: Princeton Univ. Press, 1995.

[13] E. Burstein and C. Weisbuch, Eds., Confined Electrons and Photons: New Physics and Applications. ser. NATO Advanced Science Institute Series. New York: Plenum, July 1993.

[14] N. Stefanou and A. Modinos, "Impurity bands in photonic insulators," Phys. Rev. B, vol. 57, no. 19, pp. 12 127-12133, 1998.

[15] N. W. Ashcroft and N. D. Mermin, Solid State Physics. Orlando, FL: Harcourt Brace Jovanovich, 1976.

[16] R. A. Smith, Wave Mechanics of Crystalline Solids. London, U.K.: Chapman and Hall, 1969.

[17] T. Mukaiyama, K. Takeda, H. Miyazaki, Y. Jimba, and M Kuwata-Gonokami, "Tight-binding photonic molecule modes of resonant bispheres," Phys. Rev. Lett., vol. 82, no. 23, pp. 4623-4626, 1999. 
[18] C. M. de Sterke, "Superstructure gratings in the tight-binding approximation," Phys. Rev. E, vol. 57, no. 3, pp. 3502-3509, 1998.

[19] P. R. Villeneuve, S. Fan, and J. D. Joannopoulos, "Microcavities in photonic crystals: Mode symmetry, tunability, and coupling efficiency," Phys. Rev. B, vol. 54, no. 11, pp. 7837-7842, 1996.

[20] O. J. Painter, J. Vučković, and A. Scherer, "Defect modes of a two-dimensional photonic crystal in an optically thin dielectric slab," J. Opt. Soc. Amer. B, vol. 16, no. 2, pp. 275-285, 1999.

[21] A. L. Reynolds, U. Peschel, F. Lederer, P. J. Roberts, T. F. Krauss, and P. J. I. de Maagt, "Coupled defects in photonic crystals," IEEE Trans. Microwave Theory Tech., vol. 49, p. 1860, Oct. 2001.

[22] M. Bayer, T. Gutbrod, J. P. Reithmaier, A. Forchel, T. L. Reinecke, P. A. Knipp, A. A. Dremin, and V. D. Kulakovskii, "Optical modes in photonic molecules," Phys. Rev. Lett., vol. 81, no. 12, pp. 2582-2585, 1998.

[23] M. Bayer, T. Gutbrod, A. Forchel, T. L. Reinecke, P. A. Knipp, R. Wener, and J. P. Rethmaier, "Optical demonstration of a crystal band structure formation," Phys. Rev. Lett., vol. 83, no. 25, pp. 5374-5377, 1999.

[24] A. Figotin and A. Klein, "Localization of light in lossless inhomogeneous dielectrics," J. Opt. Soc. Amer. A, vol. 15, no. 5, pp. 1423-1435, 1998.

[25] A. V. Oppenheim, A. S. Willksy, and I. T. Young, Signals and Systems. Englewood, NJ: Prentice-Hall, 1995.

[26] J. M. Wozencraft and I. M. Jacobs, Principles of Communication Engineering. New York: Wiley, 1967.

[27] A. Yariv, Optical Electronics in Modern Communications. New York: Oxford, 1997

[28] G. Watson, A Treatise on the Theory of Bessel Functions. Cambridge, U.K.: Cambridge Univ. Press, 1944.

[29] Y. R. Shen, The Principles of Nonlinear Optics. New York: Wiley, 1984.

[30] T. R. O'Meara and A. Yariv, "Time-domain signal processing via four-wave mixing in nonlinear delay lines," Opt. Eng., vol. 21, pp. 237-242, 1982.

[31] M. Segev and A. Yariv, "Phase conjugation involving incoherent counterpropagating beams in photorefractive media," Opt. Lett., vol. 16, no. 24, pp. 1938-1940, 1991.

[32] M. Bayindir, B. Temelkuran, and E. Ozbay, "Tight-binding description of the coupled defect modes in three-dimensional photonic crystals," Phys. Rev. Lett., vol. 84, no. 10, pp. 2140-2143, 2000.

[33] S. Olivier, C. Smith, M. Rattier, H. Benisty, C. Weisbuch, T. Krauss, R. Houdré, and U. Oesterlé, "Miniband transmission in a photonic crystal coupled-resonator optical waveguide," Opt. Lett., vol. 26, no. 13, pp. 1019-1021, 2001.

[34] N. G. R. Broderick and C. M. de Sterke, "Theory of grating superstructures," Phys. Rev. E, vol. 55, no. 3, pp. 3634-3647, 1997.

[35] B. J. Eggleton, C. M. de Sterke, and R. E. Slusher, "Nonlinear propagation in superstructure Bragg gratings," Opt. Lett., vol. 21, no. 16, pp. 1223-1225, 1996.

[36] C. Liu, Z. Dutton, C. H. Behroozi, and L. V. Hau, "Observation of coherent optical information storage in an atomic medium using halted light pulses," Nature, vol. 409, pp. 490-493, 2001.

[37] Y. Fainman, P.-C. Sun, and Y. T. Mazurenko, "Space-time processing with photorefractive volume holography using femtosecond laser pulses," in Photorefractive Optics: Materials, Properties, and Applications, F. Yu and S. Yin, Eds. New York: Academic, 2000, ch. 15, pp. 485-518.

[38] M. Notomi, A. Shinya, K. Yamada, J. Takahashi, C. Takahashi, and I. Yokohama, "Singlemode transmission within photonic bandgap of width-varied single-line-defect photonic crystal waveguides on SOI substrates," Electron. Lett., vol. 37, no. 5, pp. 293-295, 2001.

[39] S. G. Johnson, P. R. Villeneuve, S. Fan, and J. D. Joannopoulos, "Linear waveguides in photonic-crystal slabs," Phys. Rev. B, vol. 62, no. 12, pp. $8212-8222,2000$

[40] A. Adibi, Y. Xu, R. K. Lee, A. Yariv, and A. Scherer, "Properties of the slab modes in photonic crystal optical waveguides," J. Lightwave Technol., vol. 18, no. 11, pp. 1554-1564, 2000.

[41] M. Lončar, D. Nedeljković, T. Doll, J. Vučković, A. Scherer, and T. P. Pearsall, "Waveguiding in planar photonic crystals," Appl. Phys. Lett., vol. 77, no. 13, pp. 1937-1939, 2000.
[42] S. F. Mingaleev, Y. S. Kivshar, and R. A. Sammut, "Long-range interaction and nonlinear localized modes in photonic crystal waveguides," Phys. Rev. E, vol. 62, no. 4, pp. 5777-5782, 2000.

[43] R. S. Mackay and S. Aubry, "Proof of existence of breathers for time-reversible or Hamiltonian networks of weakly coupled oscillators," Nonlinearity, vol. 7, no. 6, pp. 1623-1643, 1994.

[44] S. John and N. Akozbek, "Nonlinear optical solitary waves in a photonic band-gap," Phys. Rev. Lett., vol. 71, no. 8, pp. 1168-1171, 1993.

[45] A. A. Sukhorukov, Y. S. Kivshar, and O. Bang, "Two-color nonlinear localized photonic modes," Phys. Rev. E, vol. 60, no. 1, pp. R41-R44, 1999.

[46] A. R. McGurn, "Intrinsic localized modes in nonlinear photonic crystal waveguides," Phys. Lett. A, vol. 251, no. 5, pp. 322-335, 1999.

[47] A. A. Sukhorukov, Y. S. Kivshar, O. Bang, and C. M. Soukoulis, "Parametric localized modes in quadratic nonlinear photonic structures," Phys. Rev. E, vol. 63, 2000.

Shayan Mookherjea (S'00) received the B.S. degree with honors in electrical engineering from the California Institute of Technology (Caltech), CA, and the S.M. degree in electrical engineering and computer science from the Massachusetts Institute of Technology (MIT), MA, in 1999 and 2000, respectively.

He is currently a doctoral candidate with the Yariv group at Caltech working on soliton-based optical communication systems and on optical information processing devices. His research interests also include optical networks, photonic crystals and periodic structures, nonlinear and quantum optics, and a wide range of topics in applied mathematics and physics.

Mr. Mookherjea is a member of Tau Beta Pi and Sigma Xi.

Amnon Yariv (S'56-M'59-F'70-LF'95) a native of Israel, received the B.S., M.S., and Ph.D. in electrical engineering from the University of California at Berkeley, CA, in 1954, 1956 and 1958, respectively.

In 1959, he went to the Bell Telephone Laboratories, Murray Hill, NJ, joining the early stages of the laser effort. In 1964, he joined the California Institute of Technology as an Associate Professor of Electrical Engineering, becoming a Professor in 1966. In 1980, he became the Thomas G. Myers Professor of Electrical Engineering and Applied Physics. In 1996, he became the Martin and Eileen Summerfield Professor of Applied Physics and Professor of Electrical Engineering. On the technical and scientific sides, he took part (with various co-workers) in the discovery of a number of early solid-state laser systems, in the original formulation of the theory of nonlinear quantum optics; in proposing and explaining mode-locked ultrashort-pulse lasers, GaAs optoelectronics; in proposing and demonstrating semiconductor-based integrated optics technology; in pioneering the field of phase conjugate optics; and in proposing and demonstrating the semiconductor distributed feedback laser. His present research efforts are in the areas of nonlinear optics, semiconductor lasers and WDM filters, especially the problem of monolithic integration of transistors, injection lasers, ultrafast $\left(<10^{-12} \mathrm{~s}\right)$ semiconductor devices and phenomena, and the propagation of optical signals in fibers, including wavelength-division multiplexing (WDM). He has published widely in the laser and optics fields and has written a number of basic texts in quantum electronics, optics, and quantum mechanics. He has received the 1980 Quantum Electronics Award of the IEEE, the 1985 University of Pennsylvania Pender Award, the 1986 Optical Society of America Ives Medal, the 1992 Harvey Prize (shared with M. Gorbachev) and the 1998 Esther Beller Medal of the Optical Society of America. He is a founder and chairman-of-the-board of ORTEL Corporation (acquired by Lucent Technologies), and a founder and a board member of Arroyo Optics Inc.

Dr. Yariv is a member of the American Physical Society, Phi Beta Kappa, the American Academy of Arts and Sciences, the National Academy of Engineering, the National Academy of Sciences, a Fellow of the Institute of Electrical and Electronics Engineers, and the Optical Society of America. 